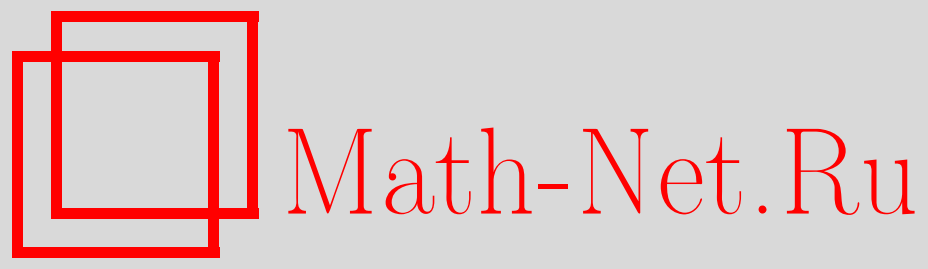

А. М. Вершик, П. П. Никитин, Описание характеров и факторпредставлений бесконечной симметрической инверсной полугруппы, Функи. анализ и его прил., 2011, том 45, выпуск 1, 16-30

DOI: https://doi.org/10.4213/faa3023

Использование Общероссийского математического портала MathNet.Ru подразумевает, что вы прочитали и согласны с пользовательским соглашением

http://www. mathnet.ru/rus/agreement

Параметры загрузки:

IP : 44.207 .124 .84

26 апреля 2023 г., 12:09:09

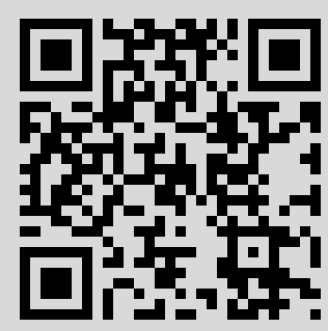




\title{
Описание характеров и факторпредставлений бесконечной симметрической инверсной полугруппы*
}

\author{
(c) 2011. А. М. ВеРшик, П. П. Никитин
}

\begin{abstract}
В статье приводится полный список неразложимых характеров бесконечной симметрической полугруппы. По сравнению с аналогичным списком для бесконечной симметрической группы необходимо ввести лишь один новый параметр, имеющий наглядный комбинаторный смысл. В работе используется теория представлений конечных симметрических полугрупп и теория представлений бесконечной симметрической группы.
\end{abstract}

\section{Введение}

Эта статья посвящена описанию характеров бесконечной симметрической полугруппы. Основной результат соединяет, с одной стороны, теорию представлений конечных симметрических полугрупп, развитую Манном [13], [14], Соломоном [17], Халверсоном [12], Вагнером [1], Престоном [16] и Поповой [11], а с другой - теорию представлений локально конечных групп, в частности, бесконечной симметрической группы, и локально полупростых алгебр, развитую в работах То́ма [18], Вершика-Керова [4]-[6], [20]. Анализ диаграммы Браттели для бесконечной симметрической полугруппы, проведенный ниже, напоминает анализ в более сложной ситуации - в задаче о характерах алгебр БрауэраВейля [7]. Симметрическая полугруппа возникала не только в литературе по теории полугрупп и их представлений, но и в связи с теорией представлений бесконечной симметрической группы [15] и для определения полугруппы кос [19]; также рассматривались $q$-аналоги симметрических полугрупп [12]. Повидимому, определение бесконечной симметрической полугруппы, данное в статье, и проблемы, связанные с ее представлениями, в литературе до сих пор не обсуждались.

Рассмотрим множество всех взаимно однозначных частичных отображений множества $\{1, \ldots, n\}$, т. е. взаимно однозначных отображений, действующих из некоторого подмножества множества $\{1, \ldots, n\}$ на некоторое (возможно, отличное от первого) подмножество множества $\{1, \ldots, n\}$. Зададим умножение двух таких отображений как композицию отображений там, где она определена. В результате мы получим полугруппу с нулем (отображение с пустой областью определения), называемую обычно симметрической инверсной полугруппой, обозначим ее $R_{n}$ (есть и другие обозначения см. [9], [17]).

Очевидным образом симметрическая группа $S_{n}$ является подгруппой полугруппы $R_{n}: S_{n} \subset R_{n}$. Полугруппа $R_{n}$ может быть представлена как полугруппа всех 0-1-матриц с не более чем одной единицей в каждой строке и каждом столбце с матричным умножением. Эта реализация аналогична натуральному пред-

*Работа выполнена при частичной поддержке грантов РФФИ-08-01-00379-а и РФФИ-0901-12175-офи-м. 
ставлению симметрической группы. Матрицы такого вида находятся во взаимно однозначном соответствии со всевозможными расстановками не бьющих друг друга ладей на $n \times n$-доске, поэтому этот моноид (полугруппа с единицей) был назван Л. Соломоном ладейным моноидом (rook monoid).

Очень важны следующие свойства инверсных полугрупп, в частности, симметрической инверсной полугруппы (см. дополнение):

(1) комплексная полугрупповая алгебра любой конечной инверсной полугруппы полупроста ([10], [14]);

(2) любая конечная инверсная полугруппа может быть изоморфно вложена в симметрическую инверсную полугруппу ([1], [16]);

(3) класс конечных инверсных полугрупп порождает в точности класс инволютивных полупростых биалгебр [2].

Следующий результат, описывающий характеры конечной инверсной полугруппы, фактически был найден несколькими авторами, его комбинаторнодинамическая характеристика приведена в [12].

Множество неприводимых представлений (и, следовательно, множество неприводимых характеров) симметрической полугруппы $R_{n}$ параметризуется набором всех диаграмм Юнга с не более чем $n$ клетками. Ветвление представлений в терминах диаграмм выглядит так: переход от неприводимого представления полугруппы $R_{n}$ к представлениям полугруппы $R_{n+1}$ состоит в том, что соответствующая представлению диаграмма Юнга либо остается без изменений, либо получает обычным образом одну новую клетку (растет).

Бесконечная симметрическая группа $S_{\infty}$ есть счетная группа всех финитных (т.е. нетождественных лишь на конечном подмножестве) взаимно однозначных отображений счетного множества в себя. Так же можно определить бесконечную симметрическую инверсную полугруппу ${ }^{1)} R_{\infty}$ как множество частичных взаимно однозначных не тождественных на конечном множестве отображений счетного множества в себя ${ }^{2)}$. Группа $S_{\infty}$ есть индуктивный предел цепочки групп $S_{n}, n=1,2, \ldots$, с естественными вложениями групп. Точно так же полугруппы $R_{n}, n=1,2, \ldots$, образуют цепочку относительно естественных мономорфизмов полугрупп ${ }^{3)} R_{0} \subset R_{1} \subset \cdots \subset R_{n} \subset \ldots$, и ее индуктивный предел есть бесконечная симметрическая инверсная полугруппа. Связь между диаграммами Браттели для бесконечной симметрической группы (граф Юнга) и диаграммой для бесконечной симметрической полугруппы естественно приводит к новой операции на графах, сопоставляющей каждой диаграмме Браттели ее «медленный» вариант. (Ср. с понятием «паскализации» графа в [7].)

Наши результаты опираются на хорошо развитую теорию представлений бесконечной симметрической группы $S_{\infty}$ и отчасти обобщают ее. Напомним, что список характеров бесконечной симметрической группы был найден Тома [18]. Новое доказательство его теоремы, данное Вершиком и Керовым [4], было основано на аппроксимации характеров бесконечной группы характерами конечных

1) Мы опускаем, как правило, слово «инверсная» и говорим просто о (бесконечной) симметрической полугруппе.

2) Тем самым в бесконечной симметрической инверсной полугруппе нулевого отображения нет, так как всякий элемент должен быть тождественным отображением на дополнении к конечному множеству.

3) При мономорфизме $R_{n} \subset R_{n+1}$ нуль полугруппы переходит не в нуль, а в некоторый проектор, точнее - в образующую $p_{n} \in R_{n+1}$, см. теорему 1.7 . 
симметрических групп и использовало комбинаторику диаграмм Юнга, которые, как хорошо известно, параметризуют неприводимые комплексные представления конечных симметрических групп. Параметрами неразложимых характеров в изложении [4] служат частоты строк и столбцов последовательности растущих диаграмм Юнга. Основной результат настоящей статьи состоит в том, что список параметров характеров бесконечной симметрической полугруппь получается из списка параметров Тома добавлением одного нового числа из отрезка [0,1]. Смысл этого параметра состоит в следующем. Параметрами неприводимых представлений конечной симметрической полугруппы $R_{n}$ также служат диаграммы Юнга, но с любым числом клеток $k$, не превосходящим $n$; последовательность растущих диаграмм, таким образом, помимо предельных частот строк и столбцов имеет еще один параметр - предел отношения $k / n$, равный относительной скорости, с которой путь в графе ветвления проходит по диаграммам Юнга, или, иначе говоря, равный замедлению скорости аппроксимации характера бесконечной полугруппы характерами конечных полугрупп.

Описание характеров полугруппы позволяет дать реализацию самого представления. Она осуществляется в том же пространстве, что и представление бесконечной симметрической полугруппы. Точнее, пространство представления строится точно так же, как и в модели факторпредставлений бесконечной симметрической группы в работе [5], но по расширенному списку параметров, см. теорему 2.16 .

В первом параграфе приведены необходимые факты теории представлений конечных симметрических инверсных полугрупп. Второй параграф посвящен непосредственно теории представлений бесконечной симметрической полугруппы $R_{\infty}$ и содержит основные результаты. В дополнении собраны общие сведения о конечных инверсных полугруппах и некоторые новые факты об их полугрупповых алгебрах как алгебрах Хопфа.

\section{§1. Теория представлений конечных симметрических инверсных полугрупп}

1.1. Полупростота полугрупповой алгебры $\mathbb{C}\left[\boldsymbol{R}_{n}\right]$. Полный список неприводимых представлений. Назовем рангом отображения $a \in R_{n}$ число элементов, на которых это отображение не определено. Каждое из множеств $A_{r}=\left\{a \in R_{n} \mid\right.$ ранг $a$ не меньше $\left.r\right\}$ при $0 \leqslant r \leqslant n$ является идеалом полугруппы $R_{n}$. Цепочка идеалов

$$
R_{n}=A_{0} \supset A_{1} \supset \cdots \supset A_{n}
$$

является главным рядом полугруппы $R_{n}$, т. е. не существует идеала, лежащего строго между $A_{r}$ и $A_{r+1}$, см. теорему 1.1 .

Обозначим через $\mathbb{C}\left[S_{n}\right]$ комплексную групповую алгебру симметрической группы $S_{n}$. Эта алгебра, как и групповая алгебра любой конечной группы, является полупростой в силу наличия инвариантного скалярного произведения.

Комплексная полугрупповая алгебра инверсной полугруппы также всегда полупроста, что следует из общей теоремы 3.3. Явное разложение алгебры $\mathbb{C}\left[R_{n}\right]$ на матричные компоненты предложено Манном [13]. 
Теорема 1.1 (Манн). Алгебра $\mathbb{C}\left[R_{n}\right]$ полупроста и имеет вид

$$
\mathbb{C}\left[R_{n}\right] \cong \bigoplus_{r=0}^{n} M_{\left(\begin{array}{l}
n \\
r
\end{array}\right)}\left(\mathbb{C}\left[S_{r}\right]\right) .
$$

Здесь $M_{l}(A)$ есть алгебра матриц порядка $l$ над алгеброй $A$. Описание представлений алгебры $\mathbb{C}\left[R_{n}\right]$ дается следующей теоремой.

Теорема 1.2 (Манн). Пусть полугруппа $S$ изоморфна полугруппе $M_{n}(G)$ матрии размера $n \times n$ с элементами из некоторой группы $G$. Пусть характеристика поля $F$ равна нулю или является простым числом, не делящим порядок группы $G$. Пусть $\left\{\gamma_{p}\right\}_{p=1}^{k}-$ полный набор неэквивалентных неприводимых представлений группы $G$ над $F$. Обозначим через $\gamma_{p}^{\prime}$ отображсене, заданное формулой

$$
\gamma_{p}^{\prime}\left(\left\{x_{i j}\right\}\right)=\left\{\gamma_{p}\left(x_{i j}\right)\right\}
$$

для любой матрицы $\left\{x_{i j}\right\} \in S=M_{n}(G)$. Тогда $\left\{\gamma_{p}^{\prime}\right\}_{p=1}^{k}-$ полный набор неэквивалентных неприводимых представлений полугруппы $S$ над $F$.

Обозначим через $\mathscr{P}_{r}$ множество всех разбиений натурального числа $r$. Из предыдущей теоремы следует, что множество неприводимых представлений полугруппы $R_{n}$ может быть естественным образом параметризовано множеством $\bigcup_{r=0}^{n} \mathscr{P}_{r}$.

Замечание 1.3. Как видно из приведенной выше формы неприводимых представлений полугруппы $R_{n}$, любое такое представление является продолжением однозначно определенного индуцированного представления группы $S_{n}$. Точнее, для неприводимого представления полугруппы $R_{n}$, отвечающего разбиению $\lambda \in \mathscr{P}_{r}$, рассмотрим представление подгруппы $S_{r} \times S_{n-r} \subset S_{n}$, где действие группы $S_{r}$ отвечает разбиению $\lambda$, а группа $S_{n-r}$ действует тривиально. Соответствующее индуцированное представление группы $S_{n}$ может быть продолжено до рассматриваемого неприводимого представления полугруппы $R_{n}$. (Это отмечено также в [15].)

Замечание 1.4. На полугрупповой алгебре симметрической полугруппы $\mathbb{C}\left[R_{n}\right]$, как и на групповой алгебре симметрической группы $\mathbb{C}\left[S_{n}\right]$, есть инволюция, переводящая, в частности, каждое неприводимое представление $\pi$ в представление $\operatorname{sgn} \pi$. Эта инволюция соответствует естественной инволюции графа Юнга и, следовательно, медленного графа Юнга (см. определение в разд. 2.1), состоящей в переходе от диаграммы к отражению этой диаграммы от диагонали. Однако эта инволюция не является инволюцией группы $S_{n}$ или полугруппы $R_{n}$.

\section{2. Формула для характеров конечной симметрической полугруп-} пы. Манну [13] принадлежит также формула, выражающая характеры симметрической инверсной полугруппы через характеры симметрических групп. Для формулировки теоремы для каждого подмножества $K \subset\{1, \ldots, n\},|K|=$ $r$, зафиксируем произвольную частичную биекцию $\mu_{K}: K \mapsto\{1, \ldots, r\}$. Через $\mu_{K}^{-}:\{1, \ldots, r\} \mapsto K$ будем обозначать отображение, обратное к $\mu_{K}$ на $K$ : $\mu_{K}^{-} \circ \mu_{K}-$ тождественное отображение на множестве $K$. 
Теорема 1.5 (Манн). Пусть $\chi^{*}-$ характер неприводимого представления полугруппы $R_{n}$, отвечающего разбиению $\lambda \in \mathscr{P}_{r}, 1 \leqslant r \leqslant n$. Пусть $\chi-$ соответствующий характер симметрической группы $S_{r}$. Тогда для любого элемента $\sigma \in R_{n}$

$$
\chi^{*}(\sigma)=\sum \chi\left(\mu_{K} \sigma \mu_{K}^{-}\right),
$$

где сумма берется по всем подмножествам $K$ области определения элемента $\sigma$, таким, что $|K|=r$ и $K \sigma=K$.

1.3. Задание полугруппы через образующие и соотношения. Нас будут интересовать семейства образующих полугрупп $\left\{R_{n}\right\}_{n=0}^{\infty}$, возрастающие при вложениях $R_{n} \subset R_{n+1}$. Этому условию удовлетворяют образующие, предложенные Поповой [11], и образующие, предложенные Халверсоном [12]. Образующие и соотношения у Халверсона выписаны для $q$-аналога симметрической инверсной полугруппы. Ниже приведен частный случай доказанного им утверждения для $q=1$.

Через $\sigma_{i}, 1 \leqslant i<n$, обозначим коксетеровские образующие группы $S_{n}$. Через $p_{i} \in R_{n}, 1 \leqslant i \leqslant n$, обозначим следующие отображения: $p_{i}(j)$ не определено, если $j \leqslant i$, и $p_{i}(j)=j$, если $j>i$.

Теорема 1.6 (Попова). Полугруппа $R_{n}$ задается образующими $\sigma_{1}, \ldots, \sigma_{n-1}$, $p_{1}$ и следуюшими соотношениями:

(1) коксетеровские соотношения для группы $S_{n}$;

(2) $\sigma_{2} p_{1} \sigma_{2}=\sigma_{2} \sigma_{3} \cdots \sigma_{n-1} p_{1} \sigma_{2} \sigma_{3} \cdots \sigma_{n-1}=p_{1}=p_{1}^{2}$;

(3) $\left(p_{1} \sigma_{1}\right)^{2}=p_{1} \sigma_{1} p_{1}=\left(\sigma_{1} p_{1}\right)^{2}$.

Теорема 1.7 (Халверсон). Полугруппа $R_{n}$ задается образующими $\sigma_{1}, \ldots$, $\sigma_{n-1}, p_{1}, \ldots, p_{n}$ и следующими соотношениями:

(1) коксетеровские соотношения для группы $S_{n}$;

(2) $\sigma_{i} p_{j}=p_{j} \sigma_{i}=p_{j}$ nрu $1 \leqslant i<j \leqslant n$;

(3) $\sigma_{i} p_{j}=p_{j} \sigma_{i}$ npu $1 \leqslant j<i \leqslant n-1$;

(4) $p_{i}^{2}=p_{i}$ nрu $1 \leqslant i \leqslant n$;

(5) $p_{i+1}=p_{i} \sigma_{i} p_{i}$ nрu $1 \leqslant i \leqslant n-1$.

Любопытное задание полугруппы $R_{n}$ образующими и соотношениями принадлежит Соломону [17]: в дополнение к коксетеровским образующим группы $S_{n}$ он рассматривает также «правый сдвиг» $\nu$ :

$$
\nu(i)= \begin{cases}i+1 & \text { при } 1 \leqslant i<n, \\ \text { не определено } & \text { при } i=n .\end{cases}
$$

Теорема 1.8 (Соломон). Полугруппа $R_{n}$ задается образующими $\sigma_{1}, \ldots$, $\sigma_{n-1}, \nu$ и следующими соотношениями:

(1) коксетеровские соотношения для группы $S_{n}$;

(2) $\nu^{i+1} \sigma_{i}=\nu^{i+1}$

(3) $\sigma_{i} \nu^{n-i+1}=\nu^{n-i+1}$;

(4) $\sigma_{i} \nu=\nu \sigma_{i+1}$

(5) $\nu \sigma_{1} \sigma_{2} \sigma_{3} \cdots \sigma_{n-1} \nu=\nu$,

где $1 \leqslant i \leqslant n-1$, кроме $n$. (4), әде $1 \leqslant i \leqslant n-2$. 


\section{§2. Теория представлений бесконечной симметрической инверсной полугруппы}

В этом параграфе предполагается знакомство с основными понятиями и фактами теории локально полупростых и конечно аппроксимативных алгебр. Кроме того, используются факты из теории представлений конечных симметрических групп $S_{n}$ и бесконечной симметрической группы $S_{\infty}$. См., например, [20].

Существует естественное вложение полугрупп $R_{n} \subset R_{n+1}$, при котором каждое отображение из полугруппы $R_{n}$ переходит в отображение из $R_{n+1}$, переводящее элемент $n+1$ в себя. Рассмотрим индуктивный предел цепочки полугрупп $R_{0} \subset R_{1} \subset \ldots \subset R_{n} \subset \ldots$, который мы будем называть бесконечной симметрической инверсной полугруппой $R_{\infty}$.

Граф ветвления алгебры $\mathbb{C}\left[\boldsymbol{R}_{\infty}\right]$. Через $\mathbb{Y}$ обозначим граф Юнга, а через $\mathbb{Y}_{n}$ - этаж этого графа, вершины которого нумеруются всеми возможными разбиениями числа $n$ (диаграммами Юнга). Через $|\lambda|$ будем обозначать число клеток диаграммы $\lambda$ (сумму частей разбиения $\lambda$ ).

Через $\widetilde{\mathbb{Y}}$ обозначим граф ветвления полугрупповой алгебры $\mathbb{C}\left[R_{\infty}\right]$. Описание графа $\widetilde{\mathbb{Y}}$ принадлежит Халверсону [12].

Теорема 2.1 (Халверсон). Граф ветвления $\widetilde{\mathbb{Y}}$ описывается следуюшим образом:

(1) вершины $n$-го этажа нумеруются всеми диаграммами Юнга с не более чем $n$ клетками, $\widetilde{\mathbb{Y}}_{n}=\bigcup_{i=0}^{n} \mathbb{Y}_{i}$;

(2) ребро соединяет вершинь $\lambda \in \widetilde{\mathbb{Y}}_{n} u \mu \in \widetilde{\mathbb{Y}}_{n+1}$, если или $\lambda=\mu$, или $\mu$ получается из $\lambda$ добавлением одной клетки.

Это приводит нас к следующему определению: медленным графом $\widetilde{\Gamma}$, построенным по графу ветвления $\Gamma$, называется следующий граф:

(1) вершины $n$-го этажа графа $\widetilde{\Gamma}$ есть объединение вершин всех этажей исходного графа $\Gamma$ с номерами не более $n, \widetilde{\Gamma}_{n}=\bigcup_{i=0}^{n} \Gamma_{i}$;

(2) ребро соединяет вершины $\lambda \in \widetilde{\Gamma}_{n}$ и $\mu \in \widetilde{\Gamma}_{n+1}$, если или $\lambda=\mu$, или вершина $\mu$ соединена ребром с вершиной $\lambda$ в исходном графе.

Напомним, что графом Паскаля $\mathbb{P}$ называется следующий граф:

(1) множество $\mathbb{P}_{n}$ вершин $n$-го этажа есть множество пар целых чисел $(n, k)$, $0 \leqslant k \leqslant n$;

(2) ребро соединяет вершины $(n, k) \in \mathbb{P}_{n}$ и $(n+1, l) \in \mathbb{P}_{n+1}$, если $l=k$ или $l=k+1$.

Заметим, что если в качестве исходного графа Г взять цепь (граф, каждый этаж которого состоит всего из одной вершины), то соответствующим медленным графом $\widetilde{\Gamma}$ будет граф $\mathbb{P}$. По аналогии с графом Паскаля мы будем нумеровать вершины $n$-го этажа $\widetilde{\Gamma}_{n}$ медленного графа парами $(n, \lambda)$, где $\lambda \in \Gamma_{i}$, $i \leqslant n$.

Замечание 2.2. Отметим, что если мы возьмем в качестве исходного графа граф Паскаля, $G=\mathbb{P}$, то медленным графом $\widetilde{G}$ будет трехмерный аналог графа Паскаля. Для трехмерного графа Паскаля медленным графом будет четырехмерный граф Паскаля и т.д. Определение многомерных аналогов графа Паскаля и описание следов соответствующих алгебр см., например, в [6]. 
Замечание 2.3. Для множества путей на графе ветвления $\widetilde{Y}$ существует биекция со случайными блужданиями на графе Юнга следующего вида: в каждый момент времени разрешено или остаться в прежней вершине, или допустимым образом спуститься на этаж ниже. Исходя из этого описания, графы, подобные $\widetilde{\mathbb{Y}}$, названы медленными.

Замечание 2.4. В работе [7] изучалась теория представлений бесконечной алгебры Брауэра. Как и в предыдущем замечании, можно построить биекцию между путями в графе ветвления алгебры Брауэра и случайными блужданиями по графу Юнга похожего вида: начиная с пустой диаграммы в графе Юнга, на каждом следующем шаге можно перейти из текущей вершины либо в вершину следующего этажа (соединенную ребром с текущей), либо в вершину предыдущего этажа (также соединенную ребром с текущей).

2.2. Сведения из теории локально полупростых алгебр. Обозначим через $T(\Gamma)$ пространство путей графа $Г$. На множестве $T(\Gamma)$ имеется «хвостовое» отношение эквивалентности (см. [4]): пути $x, y \in T(\Gamma)$ эквивалентны, $x \sim y$, если они совпадают, начиная с некоторого места. Разбиение на классы эквивалентности будем обозначать через $\xi=\xi_{\Gamma}$. Также для каждого $k \in \mathbb{N} \cup 0$ и каждого пути $s$ длины $k, s=\left(s_{0}, s_{1}, \ldots, s_{k}\right)$, обозначим через $F_{s} \subset T(\Gamma)$ цилиндр $F_{s}=\left\{t \in T \mid t_{i}=s_{i}\right.$ при $\left.0 \leqslant i \leqslant k\right\}$.

Для $x, y \in \Gamma$ обозначим через $\operatorname{dim}(x ; y)$ число путей, ведущих из вершины $x$ в вершину $y$. Через $\operatorname{dim}(y)=\operatorname{dim}(\varnothing ; y)$ обозначим число всех путей в вершину $y$. Через $\mathscr{E}(\Gamma)$ обозначим множество эргодических центральных мер на $T(\Gamma)$. Для $\mu \in \mathscr{E}(\Gamma)$ через $\mu(y)$ обозначим меру множества всех путей, проходящих через вершину $y$, т. е. суммарную меру всех цилиндров $F_{s}, s=\left(s_{0}, s_{1}, \ldots, s_{|y|}\right)$, $s_{|y|}=y$.

Мы будем использовать следующее описание характеров локально полупростой алгебры и центральных мер на ее графе ветвления (эргодический метод).

Теорема 2.5 [4]. Для любой иентральной эргодической меры $\mu$ множество путей $s=\left(s_{0}, s_{1}, \ldots, s_{f}, \ldots\right)$, таких, что для всех вериин $y$

имеет полную меру.

$$
\mu(y)=\lim _{f \rightarrow \infty} \frac{\operatorname{dim}(y) \cdot \operatorname{dim}\left(y ; s_{f}\right)}{\operatorname{dim} s_{f}},
$$

Теорема $2.6[4]$. Для любого характера ф алгебры $A=C^{*}\left(\bigcup_{f=0}^{\infty} A_{f}\right)$ существует путь $\left\{\lambda_{f}\right\}_{f=0}^{\infty}$ в диаграмме Браттели, такой, что

$$
\phi(a)=\lim _{f \rightarrow \infty} \frac{\chi_{\lambda_{f}}(a)}{\operatorname{dim} \lambda_{f}}
$$

для всех $а \in A$. Здесь $\chi_{\lambda_{f}}-$ характер представления $\lambda_{f}$ алгебри $A_{f}, \operatorname{dim} \lambda_{f}$ размерность представления.

2.3. Описание центральных мер на медленных графах. Ключевым свойством произвольного медленного графа $\widetilde{\Gamma}$ является возможность записать пространство путей $T(\widetilde{\Gamma})$ как прямое произведение пространств путей $T(\Gamma)$ и $T(\mathbb{P})$. То же верно для множества путей между любыми двумя вершинами. Более того, разбиение $\xi_{\widetilde{\Gamma}}$ и центральные эргодические меры на $T(\widetilde{\Gamma})$ также могут быть записаны в виде соответствующих произведений. 
Лемма 2.7. Пусть Г- граф некоторой локально полупростой алгебрь, $\widetilde{\Gamma}$ - соответствующий медленный граф. Тогда

1. $T(\widetilde{\Gamma})=T(\Gamma) \times T(\mathbb{P})$. Более того, число путей между любыми двумя вершинами медленного графа $\widetilde{\Gamma}$ равно произведению числа путей между соответствующими вершинами исходного графа Г и числа путей между соответствуюшими вершинами графа Паскаля $\mathbb{P}$ :

$$
\operatorname{dim}_{\widetilde{\Gamma}}\left(\left(n_{1}, \lambda_{1}\right) ;\left(n_{2}, \lambda_{2}\right)\right)=\operatorname{dim}_{\Gamma}\left(\lambda_{1}, \lambda_{2}\right) \cdot \operatorname{dim}_{\mathbb{P}}\left(\left(n_{1},\left|\lambda_{1}\right|\right) ;\left(n_{2},\left|\lambda_{2}\right|\right)\right) .
$$

2. Пусть $s_{\widetilde{\Gamma}}, t_{\widetilde{\Gamma}} \in T(\widetilde{\Gamma}), s_{\Gamma}, t_{\Gamma} \in T(\Gamma), s_{\mathbb{P}}, t_{\mathbb{P}} \in T(\mathbb{P})$ u $s_{\widetilde{\Gamma}}$ coответствует nаре $\left(s_{\Gamma}, s_{\mathbb{P}}\right), t_{\widetilde{\Gamma}}$ соответствует nаре $\left(t_{\Gamma}, t_{\mathbb{P}}\right)$. Тогда $s_{\widetilde{\Gamma}} \sim t_{\widetilde{\Gamma}}$ (относительно $\left.\xi_{\widetilde{\Gamma}}\right)$ в том и только том случае, когда $s_{\Gamma} \sim t_{\Gamma}\left(\right.$ относительно $\left.\xi_{\Gamma}\right)$ u $s_{\mathbb{P}} \sim t_{\mathbb{P}}$ (относительно $\xi_{\mathbb{P}}$ ).

Доказательство. 1. Каждому пути в графе $\widetilde{\Gamma}$ соответствует единственная строго возрастающая последовательность вершин исходного графа Г. Кроме того, каждому пути $\left(i, \lambda_{i}\right)_{i=n_{1}}^{n_{2}}$ в графе $\widetilde{\Gamma}$ можно сопоставить путь $\left(i,\left|\lambda_{i}\right|\right)_{i=n_{1}}^{n_{2}}$ в графе Паскаля. Легко понять, что по построенной паре путей исходный путь восстанавливается однозначно, откуда следует, что $T(\widetilde{\Gamma})=T(\Gamma) \times T(\mathbb{P})$.

Заметим, что построенное отображение задает биекцию между путями из вершины $\left(n_{1}, \lambda_{1}\right)$ в вершину $\left(n_{2}, \lambda_{2}\right)$ в графе $\widetilde{\Gamma}$ и парами путей - между соответствующими вершинами исходного графа Г и между соответствующими вершинами графа Паскаля $\mathbb{P}$, что доказывает формулу (1).

2. Биекция в доказательстве п. 1 строится таким образом, что для путей $t_{\widetilde{\Gamma}}=\left(t_{\Gamma}, t_{\mathbb{P}}\right)$ «хвост» пути $t_{\widetilde{\Gamma}}$ зависит только от «хвостов» путей $t_{\Gamma}$ и $t_{\mathbb{P}}$, и наоборот.

Теорема 2.8 (описание центральных мер). Существует естественная биекция $\mathscr{E}(\widetilde{\Gamma}) \cong \mathscr{E}(\Gamma) \times \mathscr{E}(\mathbb{P})$. Любая иентральная эргодическая мера $M_{\widetilde{\Gamma}} \in \mathscr{E}(\widetilde{\Gamma})$ есть произведение иентральных эргодических мер $M_{\Gamma} \in \mathscr{E}(\Gamma)$ и $M_{\mathbb{P}} \in \mathscr{E}(\mathbb{P}), a$ именно, $M_{\widetilde{\Gamma}}\left(F_{(n, \lambda)}\right)=M_{\Gamma}\left(F_{\lambda}\right) \cdot M_{\mathbb{P}}\left(F_{(n,|\lambda|)}\right)$ для любого иилиндра $F_{(n, \lambda)}$.

Доказательство. Для центральной эргодической меры $M_{\widetilde{\Gamma}} \in \mathscr{E}(\widetilde{\Gamma})$ в соответствии с разложением $T(\widetilde{\Gamma})=T(\Gamma) \times T(\mathbb{P})$ рассмотрим проекции $M_{\Gamma} \in \mathscr{E}(\Gamma)$ и $M_{\mathbb{P}} \in \mathscr{E}(\mathbb{P})$, задаваемые следующим образом:

$$
M_{\Gamma}\left(F_{\lambda}\right)=\sum_{n \geqslant|\lambda|} M_{\widetilde{\Gamma}}\left(F_{(n, \lambda)}\right), \quad M_{\mathbb{P}}\left(F_{(n, k)}\right)=\sum_{|\lambda|=k} M_{\widetilde{\Gamma}}\left(F_{(n, \lambda)}\right) .
$$

Меры $M_{\Gamma}$ и $M_{\mathbb{P}}$ центральны в силу центральности меры $M_{\widetilde{\Gamma}}$.

Далее, согласно формуле (1) из леммы 2.7 и теореме 2.5,

$$
\begin{aligned}
M_{\widetilde{\Gamma}}\left(F_{(n, \lambda)}\right) & =\lim _{f \rightarrow \infty} \frac{\operatorname{dim}\left(\left(n, \lambda_{n}\right) ;\left(f, \lambda_{f}\right)\right)}{\operatorname{dim}\left(f, \lambda_{f}\right)} \\
& =\lim _{f \rightarrow \infty} \frac{\operatorname{dim}_{\mathbb{P}}\left(\left(n,\left|\lambda_{n}\right|\right) ;\left(f,\left|\lambda_{f}\right|\right)\right)}{\operatorname{dim}_{\mathbb{P}}\left(f,\left|\lambda_{f}\right|\right)} \cdot \frac{\operatorname{dim}_{\Gamma}\left(\lambda_{n} ; \lambda_{f}\right)}{\operatorname{dim}_{\Gamma}\left(\lambda_{f}\right)} \\
& =\lim _{f \rightarrow \infty} \frac{\operatorname{dim}_{\mathbb{P}}\left(\left(n,\left|\lambda_{n}\right|\right) ;\left(f,\left|\lambda_{f}\right|\right)\right)}{\operatorname{dim}_{\mathbb{P}}\left(f,\left|\lambda_{f}\right|\right)} \cdot \lim _{f \rightarrow \infty} \frac{\operatorname{dim}_{\Gamma}\left(\lambda_{n} ; \lambda_{f}\right)}{\operatorname{dim}_{\Gamma}\left(\lambda_{f}\right)} .
\end{aligned}
$$


Пределы в правой части равенства $(2)$ существуют и равны $M_{\Gamma}\left(F_{\lambda}\right)$ и $M_{\mathbb{P}}\left(F_{(n, k)}\right)$, что доказывает требуемую формулу для $M_{\widetilde{\Gamma}}$. Эргодичность мер $M_{\Gamma}$ и $M_{\mathbb{P}}$ следует из эргодичности меры $M_{\widetilde{\Gamma}}$.

Обратно, произведение в указанном смысле центральных эргодических мер $M_{\Gamma} \in \mathscr{E}(\Gamma)$ и $M_{\mathbb{P}} \in \mathscr{E}(\mathbb{P})$ дает центральную эргодическую меру $M_{\widetilde{\Gamma}} \in \mathscr{E}(\widetilde{\Gamma})$. Центральность следует из леммы 2.7 , эргодичность - из равенства (2).

Напомним (см., например, [6]), что для графа Паскаля $\mathbb{P}$ существование пределов в теореме 2.5 равносильно тому, что для пути

$$
\left(\left(0, k_{0}\right),\left(1, k_{1}\right), \ldots,\left(f, k_{f}\right), \ldots\right)
$$

существует предел

$$
\lim _{f \rightarrow \infty} k_{f} / f=\delta, \quad \delta \in[0 ; 1],
$$

и каждому $\delta \in[0 ; 1]$ отвечает единственная центральная мера $M_{\mathbb{P}}=M_{\mathbb{P}}^{\delta}$.

Следствие 2.9. Любая мера $M_{\widetilde{\Gamma}} \in \mathscr{E}(\widetilde{\Gamma})$ параметризуется парой $\left(\delta, M_{\Gamma}\right)$, $\delta \in[0 ; 1], M_{\Gamma} \in \mathscr{E}(\Gamma)$.

Следствие 2.10. Мера $M_{\widetilde{\Gamma}}=\left(\delta, M_{\Gamma}\right)$ на $T(\widetilde{\Gamma})$ сосредоточена на путях, для которых соответствующие пути в графе Г принадлежат носителю меры $M_{\Gamma}$ и, кроме того, существует предел (3).

В частности, рассмотрим произвольную иентральную эргодическую меру $M_{\mathbb{Y}}$ на графе $\mathbb{Y}$, отвечаюшую параметрам $\alpha=\left\{\alpha_{i}\right\}, \beta=\left\{\beta_{i}\right\}, \gamma$. Тогда мера $M_{\widetilde{\mathbb{Y}}}=\left(\delta, M_{\mathbb{Y}}\right)$ на $T(\widetilde{\mathbb{Y}})$ будет сосредоточена на путях вида $\left\{\left(f, \lambda_{f}\right)\right\}$, для которых соответствующие предель для последовательности $\left\{\lambda_{f}\right\}$ равны $\left\{\alpha_{i}\right\}$ u $\left\{\beta_{i}\right\}$ и, кроме того, существует предел $\lim _{f \rightarrow \infty}\left|\lambda_{f}\right| / f=\delta$.

\section{4. Формула для характеров бесконечной симметрической полу-} группы. Приведенная выше биекция между множествами центральных мер на пространствах путей графа Г и медленного графа $\widetilde{\Gamma}$ имеет место для произвольного исходного градуированного графа Г. Эта биекция переносится на множества характеров алгебр, отвечающих этим графам (см. следствие 2.11 ниже) в силу соответствия между центральными мерами и характерами, однако сами явные формулы для характеров уже существенно зависят от графов и алгебр и не имеют универсального смысла. Ниже мы доказываем формулу, выражающую характер алгебры $\mathbb{C}\left[R_{\infty}\right]$ через соответствующий характер алгебры $\mathbb{C}\left[S_{\infty}\right]$. В этом разделе под характером мы всегда понимаем неразложимый характер.

Следствие 2.11. Описанная выше параметризация центральных мер задает биекиию, при которой каждой паре $\left(\delta, \chi_{\alpha, \beta, \gamma}^{S_{\infty}}\right)$, где $\delta \in[0,1] u \chi_{\alpha, \beta, \gamma}^{S_{\infty}}-x a$ рактер алгебры $\mathbb{C}\left[S_{\infty}\right]$, отвечает некоторый характер $\chi_{\alpha, \beta, \gamma, \delta}^{R_{\infty}}$ алгебры $\mathbb{C}\left[R_{\infty}\right]$.

Для упрощения обозначений ниже мы часто опускаем верхние индексы и параметр $\gamma$ (который выражается через $\alpha$ и $\beta$ ), полагая

$$
\chi_{\alpha, \beta} \equiv \chi_{\alpha, \beta, \gamma}^{S_{\infty}}, \quad \chi_{\alpha, \beta, \delta} \equiv \chi_{\alpha, \beta, \gamma, \delta}^{R_{\infty}} .
$$

Сопряжение элемента $\sigma \in R_{n}$ элементом симметрической группы не меняет значения характера, поэтому достаточно рассмотреть приведенные элементы $\sigma^{\circ} \in R_{n}$, для которых все неподвижные точки находятся в конце: для любого 
элемента $\sigma \in R_{n}$ существуют $g \in S_{n}, n(\sigma) \in \mathbb{N} \cup 0$, такие, что $\sigma^{\circ}=g \sigma g^{-1}$, причем $\sigma^{\circ}(i) \neq i$ при $i<n(\sigma)$ и $\sigma^{\circ}(i)=i$ при $i \geqslant n(\sigma)$. По определению вложения $R_{n} \subset R_{n+1}$ мы можем считать, что $\sigma^{\circ} \in R_{n(\sigma)}$. Порядок $n(\sigma)$ элемента $\sigma^{\circ}$ однозначно определяется элементом $\sigma$.

Введем множество $M_{k}(\sigma) \subset S_{n}$, элементы которого будем параметризовать всеми $k$-элементными подмножествами $K \subset\{1, \ldots, n\}$, отображаемыми элементом $\sigma$ на себя: каждому такому подмножеству сопоставим биекцию $\tilde{\sigma} \in S_{n}$, совпадающую с $\sigma$ на $K$ и тождественную в остальных точках.

Заметим, что для каждого элемента полугруппы $R_{n}$ существует максимальное (возможно, пустое) подмножество множества $\{1, \ldots, n\}$, взаимно однозначно отображаемое этим элементом на себя. Сужение элемента на это подмножество мы будем называть обратимой частью исходного элемента. Обратимую часть любого элемента $\sigma \in R_{n}$ можно рассматривать как элемент некоторой симметрической группы $S_{r}, r \leqslant n$, и, следовательно, можно записать как произведение непересекающихся циклов. Множество $M_{k}(\sigma)$ также может быть параметризовано множеством всех поднаборов циклов суммарной длины $k$ из циклического разложения обратимой части элемента $\sigma$.

В следующей теореме значение неразложимого характера бесконечной симметрической полугруппы на элементе $\sigma \in R_{n}$ представляется в виде линейной комбинации значений соответствующего характера Тома на каждом из элементов дизъюнктного объединения $\bigsqcup_{k} M_{k}(\sigma)$ с некоторыми коэффициентами, зависящими только от параметра $\delta$.

Теорема 2.12 (формула для характеров). Пусть $\chi_{\alpha, \beta, \gamma, \delta}^{R_{\infty}} \equiv \chi_{\alpha, \beta, \delta}-$ неразложимый характер алгебры $\mathbb{C}\left[R_{\infty}\right], \chi_{\alpha, \gamma, \beta}^{S_{\infty}} \equiv \chi_{\alpha, \beta}-$ coответствующий неразложимый характер алгебры $\mathbb{C}\left[S_{\infty}\right]$ u $\sigma \in R_{\infty}-$ приведенный элемент. Тогда

$$
\chi_{\alpha, \beta, \delta}(\sigma)=\sum_{k=0}^{n \sigma}\left(\delta^{n(\sigma)-k}(1-\delta)^{k} \cdot \sum_{\tilde{\sigma} \in M_{k}(\sigma)} \chi_{\alpha, \beta}(\tilde{\sigma})\right) .
$$

Доказательство. Согласно теореме 2.6, существует путь $\left\{\left(f, \lambda_{f}\right)\right\}_{f=0}^{\infty}$, для которого

$$
\chi_{\alpha, \beta, \delta}(\sigma)=\lim _{f \rightarrow \infty} \frac{\chi_{\left(f, \lambda_{f}\right)}^{*}(\sigma)}{\operatorname{dim}\left(f, \lambda_{f}\right)}
$$

Напомним, что элемент $\sigma \in R_{n}$ рассматривается как элемент полугруппы $R_{f}$, тождественный на подмножестве $\{n+1, \ldots, f\}$. По теореме 1.5 для вычисления характера $\chi_{\left(f, \lambda_{f}\right)}^{*}(\sigma)$ достаточно описать неподвижные под действием элемента $\sigma \in R_{f}$ подмножества множества $\{1, \ldots, f\}$ размера $\left|\lambda_{f}\right|$. Для полного описания таких подмножеств достаточно каждому неподвижному подмножеству размера $k$ множества $\{1, \ldots, n\}$ сопоставить все возможные подмножества $\left|\lambda_{f}\right|-k$ неподвижных точек из множества $\{n+1, \ldots, f\}$. Таким образом,

$$
\chi_{\left(f, \lambda_{f}\right)}^{*}(\sigma)=\sum_{k}\left(\left(\begin{array}{c}
f-n \\
\left|\lambda_{f}\right|-k
\end{array}\right) \cdot \sum_{\tilde{\sigma} \in M_{k}(\sigma)} \chi_{\lambda_{f}}(\tilde{\sigma})\right) .
$$


В силу п. 1 леммы 2.7

$$
\begin{aligned}
\chi_{\alpha, \beta, \delta}(\sigma) & =\lim _{f \rightarrow \infty} \frac{\left.\sum_{k}\left(\begin{array}{c}
f-n \\
\left|\lambda_{f}\right|-k
\end{array}\right) \cdot \sum_{\tilde{\sigma}} \chi_{\lambda_{f}}(\tilde{\sigma})\right)}{\operatorname{dim}\left(f,\left|\lambda_{f}\right|\right) \cdot \operatorname{dim}\left(\lambda_{f}\right)} \\
& =\sum_{k}\left(\lim _{f \rightarrow \infty} \frac{\left.\begin{array}{c}
f-n \\
\left|\lambda_{f}\right|-k
\end{array}\right)}{\operatorname{dim}\left(f,\left|\lambda_{f}\right|\right)} \cdot \sum_{\tilde{\sigma}} \lim _{f \rightarrow \infty} \frac{\chi_{\lambda_{f}}(\tilde{\sigma})}{\operatorname{dim}\left(\lambda_{f}\right)}\right) .
\end{aligned}
$$

Согласно следствию 2.10 и теореме 2.6 , примененной к бесконечной симметрической группе $S_{\infty}$, каждое из слагаемых в правом сомножителе правой части выражения (4) стремится к соответствующему значению характера $\chi_{\alpha, \beta}$. Кроме того, согласно следствию 2.10 , существует предел $\lim \left|\lambda_{f}\right| / f=\delta$, откуда следует, что

$$
\lim _{f \rightarrow \infty} \frac{\left(\begin{array}{c}
f-n \\
\left|\lambda_{f}\right|-k
\end{array}\right)}{\operatorname{dim}\left(f,\left|\lambda_{f}\right|\right)}=\delta^{n-k}(1-\delta)^{k},
$$

и это завершает доказательство.

Следствие 2.13. Для произвольного элемента $\sigma \in R_{n} \subset R_{\infty}$

$$
\chi_{\alpha, \beta, \delta}(\sigma)=\sum_{k=0}^{n}\left(\delta^{n-k}(1-\delta)^{k} \cdot \sum_{\tilde{\sigma} \in M_{k}(\sigma)} \chi_{\alpha, \beta}(\tilde{\sigma})\right) .
$$

Следствие 2.14. Характер $\chi_{\alpha, \beta, \delta}$ алгебры $R_{\infty}$, суженный на $S_{\infty}$, есть $\chi_{\alpha^{\prime}, \beta^{\prime}}$, где $\alpha_{1}^{\prime}=\delta, \alpha_{i}^{\prime}=(1-\delta) \alpha_{i-1}$ при $i>1 u \beta^{\prime}=(1-\delta) \beta$.

Доказательство. Мы проверим утверждение для случая $\beta=0$. Пусть $\alpha_{1}^{\prime}=$ $\delta, \alpha_{i}^{\prime}=(1-\delta) \alpha_{i-1}$ при $i>1$, и пусть $\sigma \in S_{n}$. Тогда

$$
\chi_{\alpha^{\prime}, 0}^{S_{\infty}}(\sigma)=\prod_{\gamma}\left((1-\delta)^{k_{\gamma}} \cdot \sum_{i} \alpha_{i}^{k_{\gamma}}+\delta^{k_{\gamma}}\right),
$$

где произведение берется по всем минимальным циклам $\gamma$ в циклическом разложении элемента $\sigma$ и $k_{\gamma}$ - длины этих циклов. Раскрывая произведение, получим

$$
\chi_{\alpha^{\prime}, 0}^{S_{\infty}}(\sigma)=\sum_{k} \sum_{\tilde{\sigma} \in M_{k}(\sigma)}\left((1-\delta)^{k} \delta^{n-k} \cdot \prod_{\gamma}\left(\sum_{i} \alpha_{i}^{k_{\gamma}}\right)\right),
$$

где внутреннее произведение берется по всем минимальным циклам $\gamma$ поднабора $\tilde{\sigma}$. Записывая последнее равенство в виде

$$
\chi_{\alpha^{\prime}, 0}^{S_{\infty}}(\sigma)=\sum_{k}\left(\delta^{n-k}(1-\delta)^{k} \cdot \sum_{\tilde{\sigma} \in M_{k}(\sigma)} \chi_{\alpha, 0}^{S_{\infty}}(\tilde{\sigma})\right)=\chi_{\alpha^{\prime}, 0, \delta}^{R_{\infty}}(\sigma),
$$

получаем искомое утверждение.

Замечание 2.15. В предыдущем следствии параметры $\alpha$ и $\beta$ неравноправны, несмотря на то что мы наблюдаем симметрию в графе $\widetilde{\mathbb{Y}}$. Это связано с тем, что при вложении группы $S_{n}$ в полугруппу $R_{n}$ ограничение неприводимых представлений полугруппы $R_{n}$ приводит к представлению, индуцированному с 
представления подгруппы $S_{r} \times S_{n-r} \subset S_{n}$, единичного на втором сомножителе, см. замечание 1.3. Поэтому операция ограничения представления не коммутирует с инволюцией (см. замечание 1.4), что нарушает симметрию между параметрами $\alpha$ и $\beta$.

2.5. Реализация представлений. Мы остановимся на случае $\sum_{i} \alpha_{i}=1$, т. е. все $\beta_{i}$ равны 0. Рассмотрим меру на множестве $\mathbb{N}$ вида $\mu_{\alpha}(i)=\alpha_{i}$, множество последовательностей $\mathscr{X}=\prod \mathbb{N}$ с мерой $m_{\alpha}=\prod \mu_{\alpha}$ и множество $\widetilde{\mathscr{X}}$ пар последовательностей, совпадающих с некоторого места. В пространстве $L^{2}\left(\widetilde{\mathscr{X}}, m_{\alpha}\right)$ может быть реализовано представление симметрической группы $S_{\infty}$, отвечающее параметрам $(\alpha, 0)$, см. [5], [21].

Теорема 2.16. Реализация в пространстве функиий $L^{2}\left(\widetilde{\mathscr{X}}, m_{\alpha^{\prime}}\right)$ представления группы $S_{\infty}$, отвечающего параметрам $\left(\alpha^{\prime}, 0\right)$, где $\alpha^{\prime}$ задан в следствии 2.14, может быть продолэсена до реализации представления полугруппь $R_{\infty}$, отвечающего параметрам $(\alpha, 0, \delta)$.

Доказательство. Зададим действие проектора $p_{1}$ из теоремы 1.6 следующим образом: любую последовательность $\left(a_{1}, a_{2}, a_{3}, \ldots\right) \in \mathscr{X}$ он переводит в последовательность $\left(1, a_{2}, a_{3}, \ldots\right) \in \mathscr{X}$. Соотношения теоремы 1.6 очевидным образом выполняются.

Таким образом, нам достаточно проверить, что введение дополнительного проектора не расширяет пространство представления. Но, как показано в работе [3], пространство факторпредставления симметрической группы $S_{\infty}$ совпадает со всем пространством $L^{2}\left(\widetilde{\mathscr{X}}, m_{\alpha^{\prime}}\right)$, что завершает доказательство.

Следствие 2.17. В терминах приведенной реализации можно дать короткую формулу для характеров как меры множества неподвижнны точек аналогично формуле для характеров симметрической группь (ср. [5]), а именно

$$
\chi_{\alpha, 0, \delta}(\sigma)=m_{\alpha^{\prime}}(\{x: \sigma(x)=x\}),
$$

где $\alpha^{\prime}$ задан в следствии 2.14. См. также [3].

\section{§3. Дополнение. Общие сведения о конечных инверсных полугруппах}

В этом параграфе мы преимущественно следуем монографии [9] и статье [2].

\section{1. Определение инверсной полугруппы.}

Теорема 3.1. Следующие три условия для полугруппы $S$ равносильны:

(1) для любого $a \in S$ существует $x \in S$, такой, что аха $=a$, и любъе два идемпотента полугруппь $S$ коммутируют;

(2) каждый главный левый и каждый главный правый идеал полугруппы $S$ порождается единственным идемпотентом;

(3) для любого $a \in S$ существует единственный $x \in S$, такой, что аха $=a$ $u x a x=x$.

Полугруппа, удовлетворяющая условиям теоремы 3.1, называется инверсной nолугруппой. Элементы $a$ и $x$ из п. (1) теоремы называются инверсными друг к другу, иногда используется обозначение $x=a^{-1}$. Отметим, что $(a b)^{-1}=b^{-1} a^{-1}$ для любых $a, b \in S$. 
Докажем, что симметрическая инверсная полугруппа является инверсной. Для каждого частичного отображения $\sigma \in R_{n}$, действующего из некоторого подмножества $X \subset\{1, \ldots, n\}$ в $Y \subset\{1, \ldots, n\}$, построим отображение $\sigma^{-1}$ из $Y$ в $X$, обратное в обычном смысле слова, т. е. для $y \in Y$ и $x \in X$ положим $\sigma^{-1}(y)=x$, если $\sigma(x)=y$. Элементы $\sigma$ и $\sigma^{-1}$, очевидно, инверсны друг к другу. Кроме того, идемпотентами симметрической инверсной полугруппы являются те и только те отображения, которые переводят в себя некоторое подмножество $X \subset\{1, \ldots, n\}$ и не определены на $\{1, \ldots, n\} \backslash X$. Следовательно, любые два идемпотента коммутируют, и по теореме 3.1 полугруппа является инверсной.

3.2. Аналог теоремы Кэли. Вагнер [1] и Престон [16] доказали для инверсных полугрупп аналог теоремы Кэли для групп.

Теорема 3.2. Произвольная инверсная полугруппа $S$ изоморфна инверсной подполугруппе симметрической инверсной полугруппы всех взаимно однозначных частичных отображений множества $S$.

Доказательство намного труднее, чем для группового аналога, и мы его не приводим (см. [9]). Отметим, что теорема справедлива как для конечных, так и для бесконечных инверсных полугрупп.

3.3. Полупростота полугрупповой алгебры. Для произвольной конечной полугруппы $S$ и поля $F$ можно рассмотреть полугрупповую алгебру $F[S]$ над этим полем. Базисом в алгебре $F[S]$ являются элементы полугруппы $S$, закон умножения базисных элементов совпадает с законом умножения элементов полугруппы. Необходимые и достаточные условия полупростоты алгебры $F[S]$ конечной инверсной полугруппы $S$ были независимо получены Манном [14] и Оганесяном [10].

Теорема 3.3. Полугрупповая алгебра $F[S]$ конечной инверсной полугруппы $S$ над полем $F$ полупроста тогда и только тогда, когда характеристика поля равна нулю или является простым числом, не делящим порядок никакой подеруппы из $S$.

\section{4. Инволютивные биалгебры и полугрупповые алгебры инверс-} ной полугруппы. Биалгеброй (см. [8]) мы называем векторное пространство над полем $\mathbb{C}$, в котором заданы согласованные структуры ассоциативной алгебры с единицей и коассоциативной алгебры с коединицей. А именно, должны удовлетворяться следующие эквивалентные условия:

(1) коумножение и коединица являются гомоморфизмами соответствующих алгебр;

(2) умножение и единица являются гомоморфизмами соответствующих коалгебр.

Введем также понятие ослабленной биалгебры для случая, когда выполняется только условие гомоморфности умножения и коумножения.

Групповая алгебра конечной группы со структурой свертки и диагональным коумножением является кокоммутативной биалгеброй (и даже алгеброй Хопфа). Хорошо известно (см. [8]), что полугрупповая алгебра любой конечной полугруппы с единицей (моноида) есть также кокоммутативная биалгебра с естественным определением операций. 
Инволюцией алгебры называется антилинейный антиавтоморфизм второго порядка этой алгебры, антилинейный антиавтоморфизм второго порядка коалгебры называется коинволюцией. Биалгебра, снабженная инволюцией и коинволюцией, называется инволютивной биалгеброй или биалгеброй с инволюцией, если умножение коммутирует с коинволюцией, а коумножение - с инволюцией.

В [2] показано, что класс конечных инверсных полугрупп порождает в точности класс инволютивных полупростых биалгебр.

Теорема 3.4. Полугрупповая алгебра $\mathbb{C}[S]$ конечной инверсной полугруппъ $S$ есть полупростая кокоммутативная инволютивная биалгебра. Аналогично, двойственная полугрупповая алгебра конечной инверсной полугруппь с единицей является коммутативной инволютивной биалгеброй. Обратно, всякая конечномерная полупростая кокоммутативная (в двойственном варианте коммутативная) инволютивная биалгебра изоморфна (как инволютивная биалгебра) полугрупповой алгебре (соответственно двойственной полугрупповой алгебре) конечной инверсной полугруппь с единицей.

Для инверсных полугрупп без единищы полугрупповая биалгебра является ослабленной биалгеброй (коединица не задает гомоморфизм).

\section{ЛитеРАТУРА}

[1] В. В. Вагнер, Обобщенные группь, Докл. АН СССР, 84 (1952), 24-43.

[2] А. М. Вершик, Двойственность Крейна, позитивные 2-алгебры и дилатация коумножений, Функц. анализ и его прил., 41:2 (2007), 24-43.

[3] А. М. Вершик, Несвободные действия групn и теория характеров, готовится к печати.

[4] А. М. Вершик, С. В. Керов, Асимптотическая теория характеров симметрической групnы, Функц. анализ и его прил., 15:4 (1981), 15-27.

[5] А. М. Вершик, С. В. Керов, Характеры и фактор-представления бесконечной симметрической группь, Докл. АН СССР, 257:5 (1981), 1037-1040.

[6] А. М. Вершик, С. В. Керов, Локалъно полупростые алгебры. Комбинаторная теория и $K_{0}$-бунктор, в кн.: Итоги науки и техники. Современные проблемы математики. Новейшие достижения, т. 26, ВИНИТИ, М., 1985, 3-56.

[7] А. М. Вершик, П. П. Никитин, Следъ на бесконечных алгебрах Брауэра., Функц. анализ и его прил., 40:3 (2006), 3-11.

[8] К. Кассель, Квантовые группь, Фазис, М., 1999.

[9] А. Клиффорд, Г. Престон, Алгебраическая теория полугрупn, Мир, М., 1972.

[10] В. А. Оганесян, О полупростоте системной алгебры, Докл. АН Арм. ССР, 21 (1955), 145-147.

[11] Л. И. Попова, Определяющие соотношения некоторых подгрупп частичных преобразований конечного множества, Уч. записки Ленингр. гос. пед. ин-та им. А. И. Герцена, 218 (1961), 191-212.

[12] T. Halverson, Representations of the q-rook monoid, J. Algebra, 273:1 (2004), 227251.

[13] W. D. Munn, The characters of the symmetric inverse semigroup, Proc. Cambridge Philos. Soc., 53:1 (1957), 13-18.

[14] W. D. Munn, On semigroup algebras, Proc. Cambridge Philos. Soc., 51:1 (1955), 1-15.

[15] G. Olshansky, Unitary representations of the infinite symmetric group: a semigroup approach, in: Representations of Lie Groups and Lie Algebras, Académiai Kiadó, Budapest, 1985, 181-197.

[16] G. B. Preston, Representations of inverse semi-groups, J. London Math. Soc., 29 (1954), 411-419. 
[17] L. Solomon, Representations of the rook monoid, J. Algebra, 256:2 (2002), 309-342.

[18] E. Thoma, Die unzerlegbaren, positiv-definiten Klassenfunktionen der abzählbar unendlichen symmetrischen Gruppe, Math. Z., 85:1 (1964), 40-61.

[19] V. V. Vershinin, On the inverse braid monoid, Topology Appl., 156:6, 1153-1166.

[20] A. M. Vershik, S. V. Kerov, The Grothendieck group of the infinite symmetric group and symmetric Functions (with the elements of the theory $K_{0}$-functor of AF-algebras), in: Adv. Stud. Contemp. Math., vol. 7, Gordon and Breach, New York, 1990, 39-118.

[21] A. M. Vershik, N. V. Tsilevich, On different models of representations of the infinite symmetric group, Adv. Appl. Math., 37:4 (2006), 526-540.

С.-Петербургское отделение Математического института им. В. А. Стеклова РАН e-mail: vershik@pdmi.ras.ru

С.-Петербургское отделение Математического института им. В. А. Стеклова РАН

e-mail: pnikitin0103@yahoo.co.uk 